\title{
Global Research Trends in Stem Cells for Tendon from 1991 to 2020: A Bibliometric and Visualized Study
}

Huibin Long

Capital Medical University Affiliated Beijing Friendship Hospital

\section{Ziyang Yuan}

Capital Medical University Affiliated Beijing Friendship Hospital

Heyong Yin

Capital Medical University Affiliated Beijing Friendship Hospital

\section{Bo Yang}

Capital Medical University Affiliated Beijing Friendship Hospital

Ai Guo ( $\nabla$ guoai@ccmu.edu.cn )

Capital Medical University Affiliated Beijing Friendship Hospital https://orcid.org/0000-0002-40425682

\section{Research}

Keywords: Global trends, Bibliometrics, Visualized study, Stem cell, Tendon

Posted Date: October 18th, 2021

DOl: https://doi.org/10.21203/rs.3.rs-966659/v1

License: (c) (1) This work is licensed under a Creative Commons Attribution 4.0 International License. Read Full License 


\section{Abstract}

Background: Tendinopathy is a disabling musculoskeletal disorder affecting the athletic and general populations. There have been increased studies using stem cells in treating tendon diseases. The aim of this bibliometric and visualized study is to comprehensively investigate the current status and global trends of research in stem cells for tendon.

Methods: Publications related to stem cells for tendon from 1991 to 2020 were retrieved from Web of Science. The source data were studied and indexed using a bibliometric methodology. VOS viewer software was used to conduct for the visualized study, including bibliographic coupling, co-authorship, co-citation and co-occurrence analysis and to analyze the publication trends of research in stem cells for tendon.

Results: In total, 2492 articles were included. Though the relative research interests decline since 2018, the number of publications increased annually worldwide. The United States made the largest contribution to this field, with the most publications, citations and the highest $\mathrm{H}$-index. The most contributive institutions were University of Pittsburgh, Zhejiang University, Shanghai Jiao Tong University and Chinese University of Hong Kong. The Journal of Orthopaedic Research published the most relative articles. Studies could be classified into five clusters: "animal study", "tissue engineering", "clinical study", "mechanism research" and "stem cells research", which show a trend of balanced development in this field.

Conclusions: The number of publications on stem cells in treating tendon diseases may have reached a platform based on current global trends. The United States made the largest contribution to this field. In addition, according to the inherent changes of hotspots in each cluster and the possibilities of crossresearch, the research in stem cells for tendon may exist a balanced development trend.

\section{Introduction}

Tendinopathy and tendon rupture are musculoskeletal disorders and common in clinical medicine[1]. It has been reported that tendinopathy accounts for $30 \%$ of general musculoskeletal consultations[2]. Certain tendons, such as the rotator cuff, achilles tendon, posterior tibial tendon, and patellar tendon, are more likely to cause pathological damages during the process of degeneration. In addition, the extensor and flexor tendons of the hands and fingers are often directly suffered from laceration at all ages[3].

Surgical repair is the most commonly used strategy for tendon repair, but the incompletely heeling of the tendon through surgical repair can happen on occasion. Moreover, the long-term effect of surgical repair is poor especially among the elderly due to re-rupture, restrictive adhesions, and poor strength and function related to reduced biomechanical properties[4]. Hence, non-surgical treatment, compared to the traditional surgical methods with limitations and complications, has attracted people's attention in recent years. 
Some approaches, including rest, usage of non-steroidal anti-inflammatory drugs, cryotherapy, injection therapy with corticosteroids, laser therapy, continuous passive motion and restrictive bracing, have been well recognized as the effective way of controlling pain and to reduce the inflammation and swelling of the injured tissue[5-7]. However, corticosteroids as well as non-steroidal anti-inflammatory drugs, can only alleviate pain and inflammation but has no function in tendon/ligament healing[8-10].

Recently, stem cells are considered to be effective to promote tendon healing. A series of studies have reported the healing ability from different sources of stem cells[11-13], inspiring further researches to investigate the underling mechanism. To provide an overview of research trends and promote better research development, it is needed to summarize the current status of stem cells research in tendon. Nevertheless, the global development trend regarding stem cells for tendon has not been well studied yet.

Publication is the critical part of scientific research which could be regarded as a significant indicator of research contribution[14]. Based on the literature databases and literature metrology characteristics, bibliometric analysis can provide information about the development of a certain field and compare the contributions of institutions, countries, and journals, which can qualitatively and quantitatively evaluate trends in research activity over time[15]. Bibliometric analysis has also been applied in developing policy and clinical practice guidelines[16]. The aim of our study was to evaluate the current status and trends in terms of stem cells research in tendon.

\section{Materials And Methods}

\section{Data source}

Bibliometric analysis was performed based on the Science Citation Index-Expanded (SCI-E) and Social Science Citation Index (SSCl) of the Web of Science (WoS), which are considered as the first-rank database for bibliometrics, covering more than 12,000 international scientific journals with the most impact and the highest quality[17].

\section{Search strategy}

All publications were searched in WoS from database inception to 31 December, 2020, which included the articles in this field over the past 30 years. In the present study, the search terms were as follows: theme $=$ stem cell ${ }^{*}$ AND theme $=$ tendon AND publishing year $=(1991-2020)$ AND Language $=($ English $)$ AND Document types $=$ (Article OR Review). We also refined the search for certain countries or regions by indexing country/region in the WoS. Retrieval work was performed in the same day (on September 25, 2021) to avoid the variations due to daily updates. Informed consent was not required because the data is all secondary data without any personal information.

\section{Data collection}


The full records of all eligible publications including title, authors, year of publication, nationalities, affiliations, journal, keywords and abstract, were downloaded as TXT files from the WoS database. Two authors (HB Long and ZY Yuan) independently screened and extracted the data entry and collection. Any disagreement was resolved by discussion for reaching a consensus. Finally, the two authors manually verified and inputted the data in Microsoft Excel 2019 and further analyzed the data with GraphPad Prism 9 separately.

\section{Bibliometric analysis}

The basic characteristics of publications were described using the intrinsic function of WoS. H-index was a useful measure to evaluate the impact of scientific study, and shows that a scientist or scholar has published $\mathrm{H}$ papers and each of which has been cited in other publications at least $\mathrm{H}$ times. As a result, the $\mathrm{H}$-index reflects both the number of publications and the number of citations per publication[18, 19$]$. Relative research interest (RRI) was defined as the number of publications in a certain field divided by allfield publications per year. R software (version 4.0.2, R core team) was used to draw the time curve of publications using the logistic regression model: $f(x)=c /\left[1+a \times \exp ^{(-b \times(x-1991))}\right]$. In this formula, the independent variable $x$ refers to the year and the $f(x)$ refers to the corresponding number of publications. The formula $T=$ Ina/b +1991 was used to calculate the inflection point defined as the time when the growth rate of publications changes from positive to negative.

\section{Visualized analysis}

VOS viewer (Leiden University, Leiden, The Netherlands) was used for the visualized analysis of the publications[17]. In the present study, VOS viewer was used for bibliographic coupling, co-authorship, cocitation and co-occurrence analyses.

\section{Results}

\section{Global publication trends}

\section{Number of global publications}

In total, 2492 publications met the criteria. From 1991 to 2020 . Though the RRI declined since 2018, there has been a steadily increasing trend of global publications annually. The number of publications increased from 2 (1991) to 250 (2020). Most research was published in 2019 (255, 10.23\%) (Fig. 1A).

\section{Contributions of countries and regions}


A total of 65 countries and regions published articles in this domain. The United States published the most papers $(938,37.64 \%)$, followed by China $(567,22.75 \%)$, England $(219,8.79 \%)$ and Germany (195, 7.83\%). (Fig. 1B and C).

\section{Global trends of publications}

The logistic regression model was used to construct the time curve of the number of the publications, which could help to predict the future trend in the next few years. Figure 1D showed the model fitting curves of the growth trend. According to the time curve, the number of publications in this field was estimated to reach a platform.

\section{Quality of the publications}

Papers from the United States had the highest citation frequency $(68,195)$. Then, China ranked second in total citation frequency $(14,776)$, followed by England $(12,132)$, Germany $(5,657)$ and Japan $(5,266)$ (Fig. $2 A)$. Fig. $2 B$ showed the average citation frequency of top 20 countries. Wales had the highest average citation frequency (197.33), followed by Norway (88.2) and Singapore (87.36). Besides, United States outranked other countries with H-index of 103, followed by China (57), England (50), Germany (40) and Japan (39) (Fig. 2C).

\section{Analysis of global publication}

\section{Journals}

The Journal of Orthopaedic Research published the most articles, with 100 publications. American Journal of Sports Medicine ranked second with 83 articles. Moreover, there were 77 articles in Tissue Engineering Part $A$ and 63 articles in Biomaterials on the research of stem cells for tendon. The top 20 journals with most papers were presented in Fig. 3A.

\section{Research orientation}

Figure 3B showed the top 20 research orientations related to stem cells for tendon. The top 5 popular areas of research were cell biology (747 papers), orthopedics (514 papers), engineering (487 papers), materials science (443 papers) and research experimental medicine (258 papers), respectively.

\section{Authors}

The top 20 authors published a total of 637 papers, which accounted for $25.56 \%$ of all articles in this field (Fig. 3C). Ouyang HW from China published the most researches on stem cells for tendon with 47 papers, 
followed by Chen X with 41 papers and Yin Z with 40 papers. In this study, we included all authors into the analysis, regardless of the relative contributions of the authors to a single study.

\section{Institution output}

The top 20 contributive institutions with the most articles were shown in Fig. 3D. The University of Pittsburgh published the most articles, with 96 papers. Zhejiang University ranked second (70 papers), followed by the Shanghai Jiao Tong University and Chinese University of Hong Kong (both 64 papers).

\section{Bibliographic coupling analysis}

Compared with other measurement of citation analysis, bibliographic coupling has advantages to construct a similarity relationship between documents. Bibliographic coupling will happen when two works cite a common third one in their bibliographies, indicating that the two works share a related theme[20].

\section{Journals}

We used VOS viewer to analyze the names of journals in total publications. As illustrated in Fig. 4A, 113 journals appeared in the bibliometric map (the minimum number of publications of each journal was over five). The top five journals with greatest total link strengths were as follows: Tissue Engineering Part $A$ (13,345 times), Journal of Orthopaedic Research (13,320 times), Biomaterials (10,755 times), American Journal of Sports Medicine (9,011 times) and Stem Cells International (8,993 times).

\section{Institutions}

There were 265 institutions included (the minimum number of publications of an institution was over five) and their publications were analyzed by VOS viewer (Fig. 4B). The top five institutions with greatest total link strengths were as follows: Chinese University of Hong Kong (17,189 times), University of Pittsburgh (16,529 times), Zhejiang University (15,872 times), Shanghai Jiao Tong University $(10,824$ times), and University of Connecticut (8,026 times).

\section{Countries and regions}

There were 32 countries and regions included (the minimum number of publications from a country or region was over five) and their publications were also analyzed by VOS viewer (Fig. 4C). The top five countries or regions with greatest total link strengths were as follows: United States (460,795 times), China (343,358 times), England (201,947 times), Germany (175,086 times), and Italy (153,702 times). 


\section{Co-authorship analysis}

Co-authorship analysis presents the relationship of items which was built according to the number of coauthored documents, which acts as an effective role in evaluating leading scientists, countries, and organizations[21].

\section{Authors}

378 authors were identified (the minimum number of publications from an author was over five) and their publications were analyzed by VOS viewer (Fig. 5A). The top five authors with greatest total link strengths were as follows: Chen X (262 times), Yin Z (245 times), Heng BC (181 times), Shen WL (129 times), and Tang KL (122 times).

\section{Institutions}

Studies (defined as minimum number of documents form an organization that were used more than five) identified in the 265 institutions were analyzed by VOS viewer (Fig.5B). The top five institutions with greatest total link strengths were as follows: Chinese University of Hong Kong (86 times), Zhejiang University (81 times), Harvard University (73 times), University of Pittsburgh (70 times) and Columbia university (66 times).

\section{Countries and regions}

The studies in 32 identified countries and regions (the minimum number of studies from a country or region was over five) were analyzed by VOS viewer (Fig. 5C). The top five countries and regions with greatest total link strengths were as follows: United States (337 times), China (210 times), England (152 times), Germany (140 times), and Italy (104 times).

\section{Co-citation analysis}

Co-citation analysis indicates the relationship of items which are built based upon the number of times they were cited together, and it has abilities to avoid academic isolation and to accelerate knowledge integration for consistency of cross discipline[22].

\section{Journals}

The journal was included if the minimum number of citations from a source was over 20 . There were 825 journals met the criteria (Fig. 5D). The top five journals with greatest total link strengths were as follows: Biomaterials (554,821 times), Journal of Orthopaedic Research (429,514 times), American Journal of 
Sports Medicine (385,081 times), Journal of Bone and Joint Surgery-American volume (282,060 times) and Tissue Engineering Part $A$ (242,487 times).

\section{Publications}

A total of 808 publications (the minimum number of citations of a reference was over 20) were analyzed using VOS viewer (Fig. $5 \mathrm{E}$ ). The top five publications with greatest total link strengths were as follows: $\mathrm{Bi}$ et al.[23] (9936 times); Schweitzer et al.[24] (5733 times); Pittenger et al.[25] (4522 times); Young et al.[26] (4356 times) and Awad et al.[27](4314 times).

\section{Co-occurrence analysis}

Co-occurrence analysis identifies that the relationship of items is constructed based upon the number of publications when they occur together. It is a powerful tool to evaluate research areas as well as hot issues, and to track the academic progress[28]. The keywords with minimum number of occurrences over five, were analyzed by VOS viewer. As illustrated in Fig. 6A, the 213 identified keywords could be divided into five clusters: "animal study", "tissue engineering", "clinical study", "mechanism research" and "stem cells research".

In the "animal study" cluster, the most used keywords were mesenchymal stem-cells, regeneration, anterior cruciate ligament and patellar tendon. In the cluster of "tissue engineering", the primary keywords were tendon, in-vitro, tissue engineering and tissue. In the "clinical study", the main keywords were repair, platelet-rich plasma, achilles tendon and tendinopathy. In the "mechanism research" cluster, the frequently used keywords were differentiation, stem cells, expression and extracellular-matrix. As for the cluster of "stem cells research", the main keywords were bone marrow, stromal cells, marrow stromal cells and mesenchymal stem cells. These results showed the research field distribution of publications related to the study of stem cells for tendon.

In Fig.6B, VOS viewer applied different colors to keywords based on when they appeared in literature for the average time. The purple color indicated that the keywords appeared earlier while keywords in yellow color appeared later. Generally, each of the 5 clusters had a trend of balanced development. Both of 5 clusters were undergoing different degrees of changes on the research hotspot, which meant a diversified developing trend. Some keywords, such as tenogenic differentiation belonged to the "tissue engineering" cluster, had potential to be studied more in future.

\section{Discussion}

\section{Global trends of research in stem cells for tendon}


Bibliometric and visualized research can be used to show the current status and predict the future development trends in research. Thus, the study aimed to evaluate the status and global trends of stem cells for tendon with respect to publications, contributing countries, institutions and research orientations. As shown in this study, number of publications increased with time, the RRIs increased over time until 2017 and then began to decrease gradually. According to the current data, a platform of studies on this field might be published in the next few years.

\section{Status and quality of global publications}

The H-index and total number of citations represent the academic influence and publication quality of a country[29]. The United States was the leading country in the total number of publications and total citation frequency, as well as the $\mathrm{H}$-index. China was regarded as a vital role which made abundant contribution to this field in terms of total number of publications, total citation frequency and H-index. Some countries, including Wales and Norway, have also played an important role because of their high average citation frequencies. Journal of Orthopaedic Research, American Journal of Sports Medicine, Tissue Engineering Part $A$ and Biomaterials published the most studies on the research of stem cells for tendon. The listed journals have great possibilities to propose the latest academic findings in this field (Fig. 4A). Almost all the top 20 institutions come from the top 5 countries with the largest number of publications, meaning that the academic level of a country mainly depends on the number of its highquality research institutions. Ouyang HW, Chen $X$ and $Y$ in $Z$ were the top three authors who published the most articles in this field. The top 20 authors, listed in the Fig. 3B, may have a substantial impact on the development and lead the newest advancement in this field.

On the other hand, the association between papers with respect to country, institution and journal was evaluated by bibliographic coupling analysis in this study. After bibliographic coupling analysis, information about how authors use and build links based on the existing literature was provided. Our study showed that Tissue Engineering Part $A$ was the most closely related journal and Chinese University of Hong Kong published the most relevant articles. As for countries, the United States lied in the leading position in this field. Co-authorship analysis was performed to assess collaboration among countries, institutions and authors. The one with greater total link strength suggested that the author/institution/country would be more likely to has cooperation with others. In our study, the results of co-authorship analysis were Chen X, Chinese University of Hong Kong, the United States, respectively. Cocitation analysis was conducted to evaluate the academic impact of studies, by counting the number of times cited together. The landmark studies with larger total co-citation frequency could be regarded as the indicator in this field. Biomaterials was the journal with the highest citation frequency in this domain.

\section{Research focus on the stem cells for tendon}

Using the co-occurrence analysis, the research directions and hotspots in this field were identified. The map of the co-occurrence network was constructed based on the keywords of all included studies. As 
shown in Fig. 6A, five research orientations were discovered, including "animal study", "tissue engineering", "clinical study", "mechanism research" and "stem cells research". This result helps establish certain domains for further investigation. In the co-occurrence map, the keywords, including mesenchymal stem-cells, tendon, repair, differentiation and in-vitro were highlighted with bigger icons. Thus, further high-quality studies concentrating on the mesenchymal stem-cells for tendon repair in these five clusters may still needed to deeply investigate.

The overlay visualization map was color established by VOS viewer, with different color compared to the map of the co-occurrence network, based on the average time the keywords appeared in the papers[14, 30]. This method was used to monitor the directions of research. In this overlay visualization (Fig. 6B), the color bar indicated the year of publication. Nevertheless, the density of each color node (from purple to yellow) in the five clusters was evenly distributed, which showed that the five research directions had a balanced development trend. In addition, each direction itself was also undergoing changes in research hotspots, showing a diversified development trend.

Based on the results of our study, the bibliometric and visualized analysis demonstrate encouraging information of stem cells in the treatment of tendon diseases, suggesting that there exist broad research prospects and the feasibility of in-depth research. Using the bibliometric and visualized analysis, researchers can have overall knowledge on the leading countries, authors, and institutions in this field. With the above information, it is easier for investigators to obtain valuable information according to their own requirement. Additionally, based on the co-occurrence analysis and overlay visualization map, researchers could clarify the hot spots and efficiently determine the specific direction of in-depth research, funding agencies could also make more rational investment plan in stem cells for tendon.

\section{Strengths and limitations}

The present study provided a comprehensive perspective of the worldwide status and trends of studies about the stem cells for tendon using bibliometric and visualized analyses. However, there were still a few limitations which are inevitable. In this study, we performed bibliometric analysis based on WoS as database. Other major databases, such as Pubmed, Embase and Cochrane library were abandoned, which caused data source bias. Secondly, we only chose English language studies based on WoS, other non-English language papers may have been neglected, especially Chinese. Since China has large population of tendinopathy patients and acts as an important role with huge contribution in the field of research in stem cells for tendon, excluding non-English publications may result in language bias. Additionally, some recently published papers with high quality, might not be paid enough attention due to the absence of high citation frequency which require long time to achieve. Therefore, there was still need to focus on the latest primary studies and other non-English studies in our daily research work.

\section{Conclusions}


This study showed the current status and global trends in stem cells for tendon. The United States was the leading country in contribution and influence in this field. The number of publications on stem cells in treating tendon diseases may have reached a platform based on current global trends. Furthermore, there may be a trend of balanced development in this field, with the potential for internal changes in hot spots in various sub-directions of the research.

\section{Abbreviations}

WoS: Web of Science; SCIE: Science Citation Index-Expanded; SSCI: Social Science Citation Index; RRI: Relative research interest.

\section{Declarations}

\section{Ethics approval and consent to participate}

Not applicable.

\section{Consent for publication}

Not applicable.

\section{Availability of data and materials}

The datasets used and/or analyzed during the current study are available from the corresponding author on reasonable request.

\section{Competing interests}

The authors declare that there are no conflicts of interest regarding the publication of this paper.

\section{Funding}

This work was supported by the Beijing Postdoctoral Research Foundation of China (Grant Number: 2021-ZZ-010), the funders had no role in study design, data collection, analysis and interpretation, decision to publish, or preparation of the manuscript.

\section{Authors' contributions}


$H L, Z Y, B Y$ and $A G$ conceived and designed the study. HL and ZY collected, validated and analyzed data. ZY drafted the primary manuscript. HL created the time curve of the publications by logistic regression model and distribution of publications in world map. $\mathrm{HL}, \mathrm{HY}, \mathrm{BY}$, and $\mathrm{AG}$ made contribution to the manuscript revision. All authors have read and approved the final manuscript.

\section{Acknowledgments}

The authors are grateful to Jiaxiang Gao for statistical input into the database and the regression model, and Dan Xing for discussions and support on the project.

\section{References}

1. Andarawis-Puri N, Flatow EL, Soslowsky LJ. Tendon basic science: Development, repair, regeneration, and healing. J Orthop Res. 2015 Jun;33(6):780-4.

2. Millar NL, Murrell GA, McInnes IB. Inflammatory mechanisms in tendinopathy - towards translation. Nat Rev Rheumatol. 2017 Jan 25;13(2):110-22.

3. Docheva D, Muller SA, Majewski M, Evans CH. Biologics for tendon repair. Adv Drug Deliv Rev. 2015 Apr;84:222-39.

4. Yan Z, Yin H, Nerlich M, Pfeifer CG, Docheva D. Boosting tendon repair: interplay of cells, growth factors and scaffold-free and gel-based carriers. J Exp Orthop. 2018 Jan 5;5(1):1.

5. Paul WA. Chapter 4 - Tendinopathy I: Understanding Epidemiology, Pathology, Healing, and Treatment. 2015:113-47.

6. Rodrigues MT, Reis RL, Gomes ME. Engineering tendon and ligament tissues: present developments towards successful clinical products. J Tissue Eng Regen Med. 2013 Sep;7(9):673-86.

7. Siegel L, Vandenakker-Albanese C, Siegel D. Anterior cruciate ligament injuries: anatomy, physiology, biomechanics, and management. Clinical journal of sport medicine: official journal of the Canadian Academy of Sport Medicine. 2012 Jul;22(4):349-55.

8. Chan KM, Fu SC. Anti-inflammatory management for tendon injuries - friends or foes? Sports medicine, arthroscopy, rehabilitation, therapy \& technology: SMARTT. 2009 Oct 13;1(1):23.

9. Ziltener JL, Leal S, Fournier PE. Non-steroidal anti-inflammatory drugs for athletes: an update. Annals of physical and rehabilitation medicine. 2010 May;53(4):278-82, 82-8.

10. Connizzo BK, Yannascoli SM, Tucker JJ, Caro AC, Riggin CN, Mauck RL, et al. The detrimental effects of systemic Ibuprofen delivery on tendon healing are time-dependent. Clin Orthop Relat Res. 2014 Aug;472(8):2433-9.

11. Huang $Y, H e B$, Wang $L$, Yuan B, Shu H, Zhang F, et al. Bone marrow mesenchymal stem cell-derived exosomes promote rotator cuff tendon-bone healing by promoting angiogenesis and regulating $\mathrm{M} 1$ macrophages in rats. Stem Cell Res Ther. 2020 Nov;25(1):496. 11(. 
12. Madhurakkat Perikamana SK, Lee J, Ahmad T, Kim EM, Byun H, Lee S, et al. Harnessing biochemical and structural cues for tenogenic differentiation of adipose derived stem cells (ADSCs) and development of an in vitro tissue interface mimicking tendon-bone insertion graft. Biomaterials. 2018 May;165:79-93.

13. Li K, Deng Y, Deng G, Chen P, Wang Y, Wu H, et al. High cholesterol induces apoptosis and autophagy through the ROS-activated AKT/FOXO1 pathway in tendon-derived stem cells. Stem Cell Res Ther. 2020 Mar;20(1):131. 11(.

14. Xing D, Zhao Y, Dong S, Lin J. Global research trends in stem cells for osteoarthritis: a bibliometric and visualized study. Int J Rheum Dis. 2018 Jul;21(7):1372-84.

15. Zhang Q, Zhao Y, Xing D, Lin J. Global Research Trends in Shock Wave for Therapy from 1990 to 2019: A Bibliometric and Visualized Study. Biomed Res Int. 2021;2021:3802319.

16. Avcu G, Sahbudak Bal Z, Duyu M, Akkus E, Karapinar B, Vardar F. Thanks to Trauma: A Delayed Diagnosis of Pott Disease. Pediatric emergency care. 2015 Dec;31(12):e17-8.

17. Aggarwal A, Lewison G, Idir S, Peters M, Aldige C, Boerckel W, et al. The State of Lung Cancer Research: A Global Analysis. Journal of thoracic oncology: official publication of the International Association for the Study of Lung Cancer. 2016 Jul;11(7):1040-50.

18. Bornmann L, Daniel HD. The state of $\mathrm{h}$ index research. Is the $\mathrm{h}$ index the ideal way to measure research performance? EMBO reports. 2009 Jan;10(1):2-6.

19. Bertoli-Barsotti L, Lando T. A theoretical model of the relationship between the h-index and other simple citation indicators. Scientometrics. 2017;111(3):1415-48.

20. Biscaro C, Giupponi C. Co-authorship and bibliographic coupling network effects on citations. PloS one. 2014;9(6):e99502.

21. Fonseca Bde P, Sampaio RB, Fonseca MV, Zicker F. Co-authorship network analysis in health research: method and potential use. Health research policy and systems. 2016 Apr 30;14(1):34.

22. Trujillo CM, Long TM. Document co-citation analysis to enhance transdisciplinary research. Science advances. 2018 Jan;4(1):e1701130.

23. Bi Y, Ehirchiou D, Kilts TM, Inkson CA, Embree MC, Sonoyama W, et al. Identification of tendon stem/progenitor cells and the role of the extracellular matrix in their niche. Nature medicine. 2007 Oct;13(10):1219-27.

24. Schweitzer R, Chyung JH, Murtaugh LC, Brent AE, Rosen V, Olson EN, et al. Analysis of the tendon cell fate using Scleraxis, a specific marker for tendons and ligaments. Development (Cambridge, England). 2001 Oct;128(19):3855-66.

25. Pittenger MF, Mackay AM, Beck SC, Jaiswal RK, Douglas R, Mosca JD, et al. Multilineage potential of adult human mesenchymal stem cells. Science (New York, NY). 1999 Apr 2;284(5411):143-7.

26. Young RG, Butler DL, Weber W, Caplan Al, Gordon SL, Fink DJ. Use of mesenchymal stem cells in a collagen matrix for Achilles tendon repair. J Orthop Res. 1998 Jul;16(4):406-13. 
27. Awad HA, Boivin GP, Dressler MR, Smith FN, Young RG, Butler DL. Repair of patellar tendon injuries using a cell-collagen composite. J Orthop Res. 2003 May;21(3):420-31.

28. Gao Y, Wang Y, Zhai X, He Y, Chen R, Zhou J, et al. Publication trends of research on diabetes mellitus and T cells (1997-2016): A 20-year bibliometric study. PloS one. 2017;12(9):e0184869.

29. Bastian S, Ippolito JA, Lopez SA, Eloy JA, Beebe KS. The Use of the h-Index in Academic Orthopaedic Surgery. The Journal of bone and joint surgery American volume. 2017 Feb 15;99(4):e14.

30. Hesketh KR, Law C, Bedford H, Hope S. Co-Occurrence of Health Conditions during Childhood: Longitudinal Findings from the UK Millennium Cohort Study (MCS). PloS one. 2016;11(6):e0156868.

\section{Figures}

A

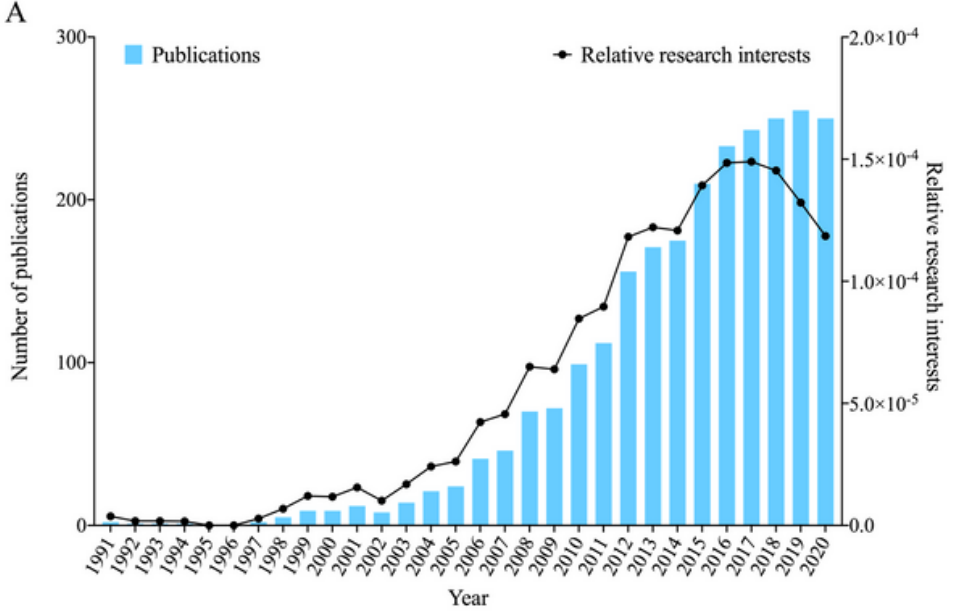

C

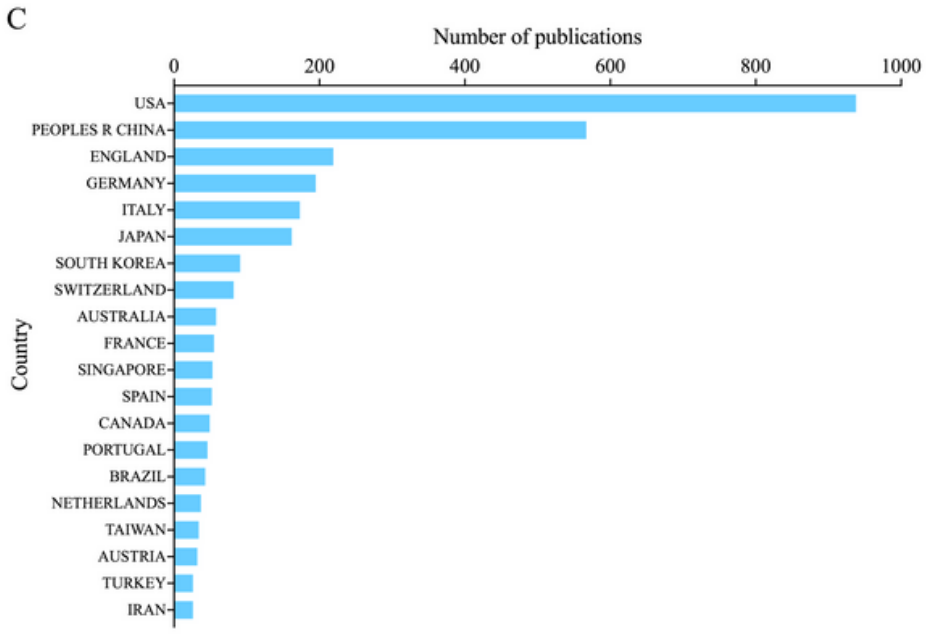

B
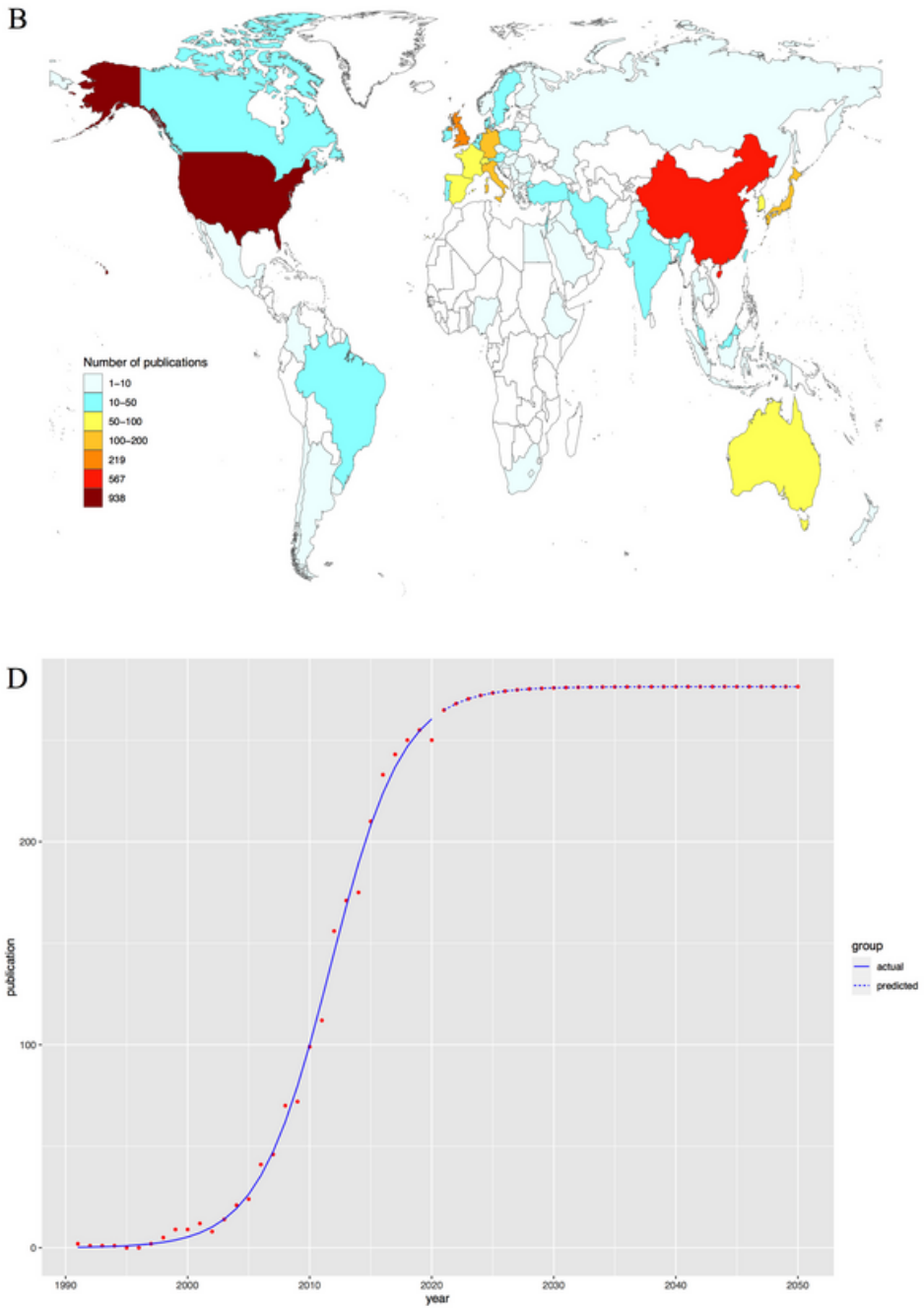

Figure 1

A The total number and relative research interests of publications related to the publications of stem cells for tendon. The blue bars indicate the number of publications each year, and the black curve means the relative research interests. B Distribution of the publications of stem cell for tendon in world map. C The top 20 countries of total number of publications. D Model fitting curves of trends in global publications. 
A

Sum of times cited

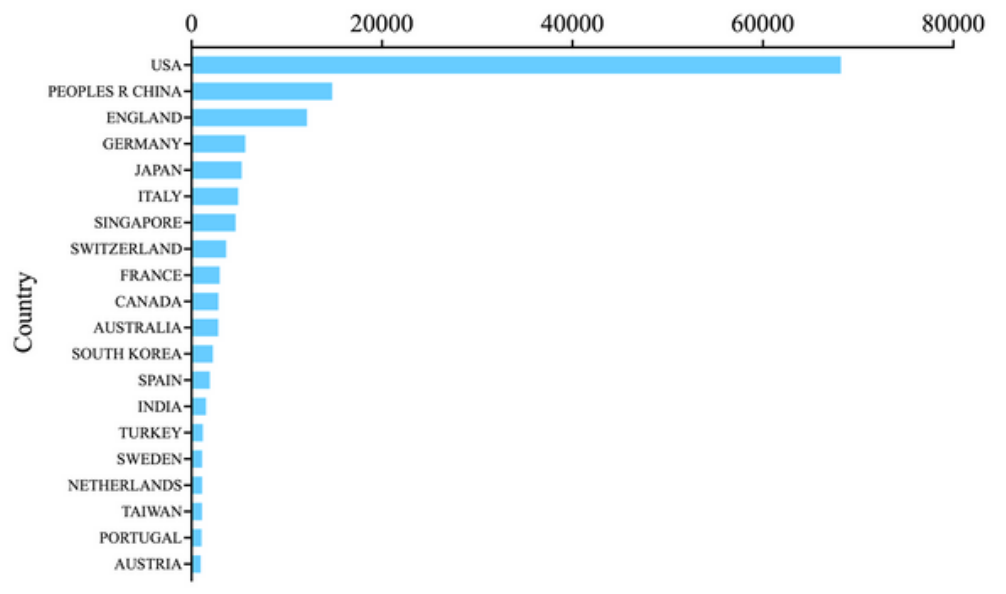

B Average citations per item

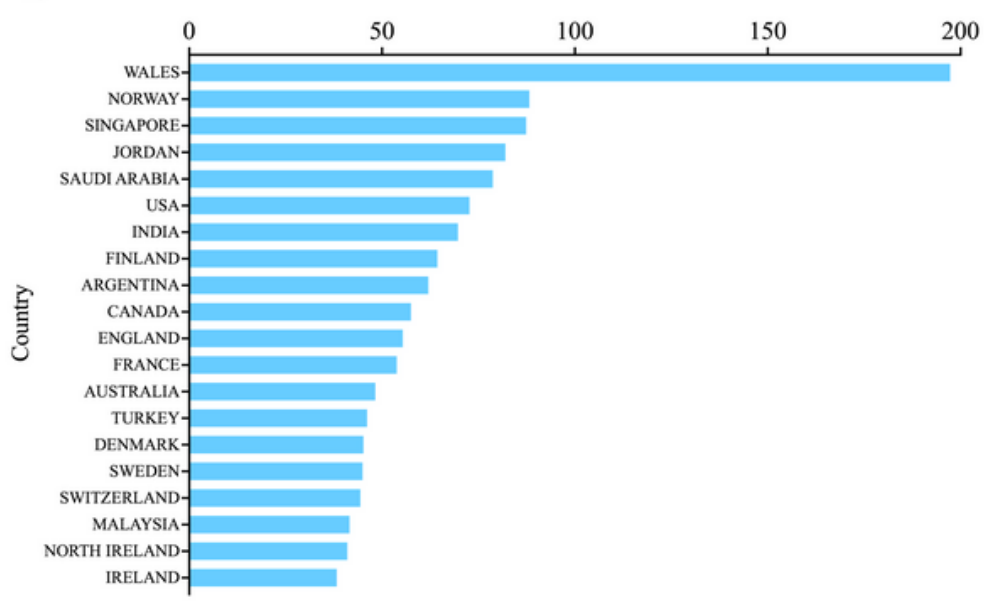

C

H-index

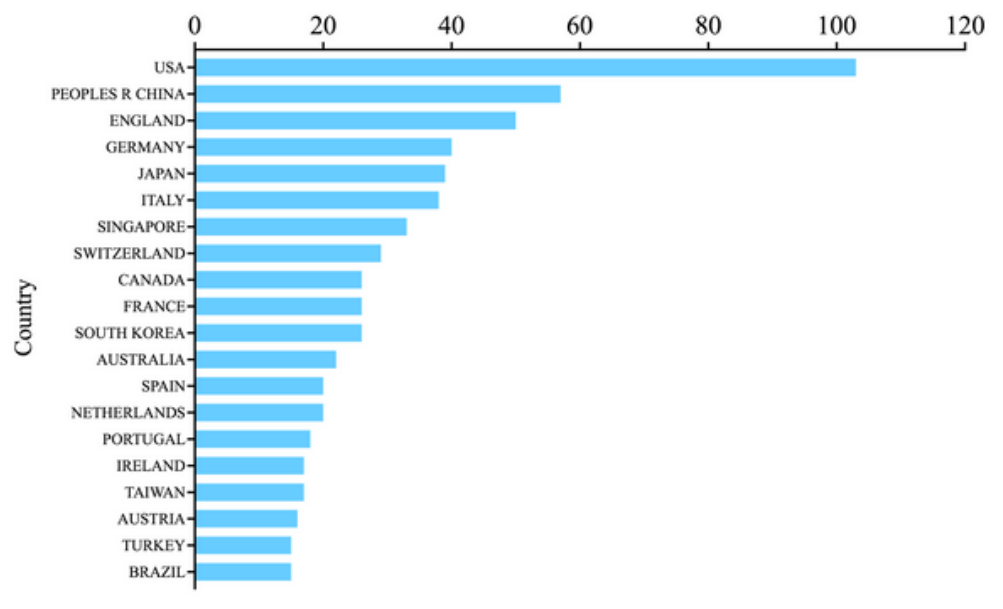

\section{Figure 2}

A The top 20 countries of total citations. B The top 20 countries of average citations per paper. C The top 20 countries of $\mathrm{H}$-index of publications. 
A
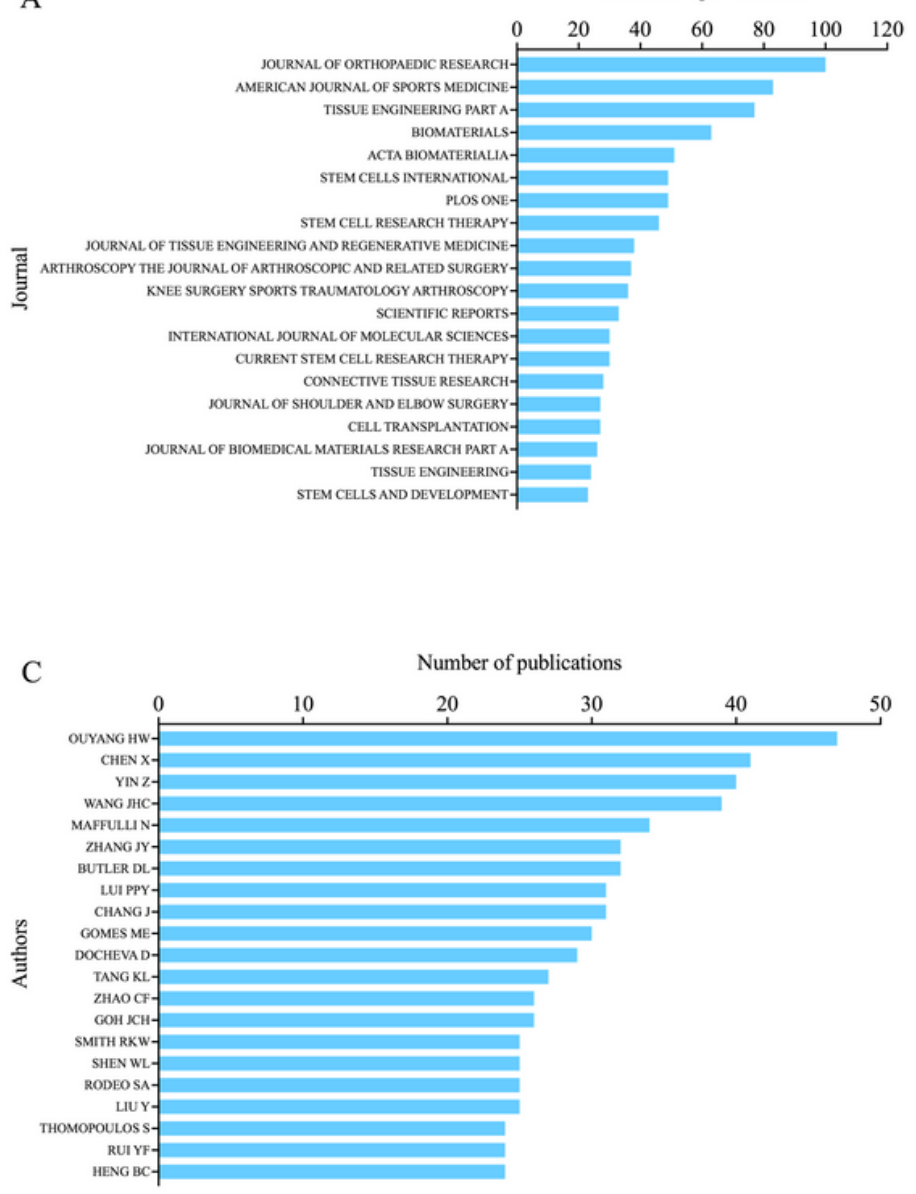

B

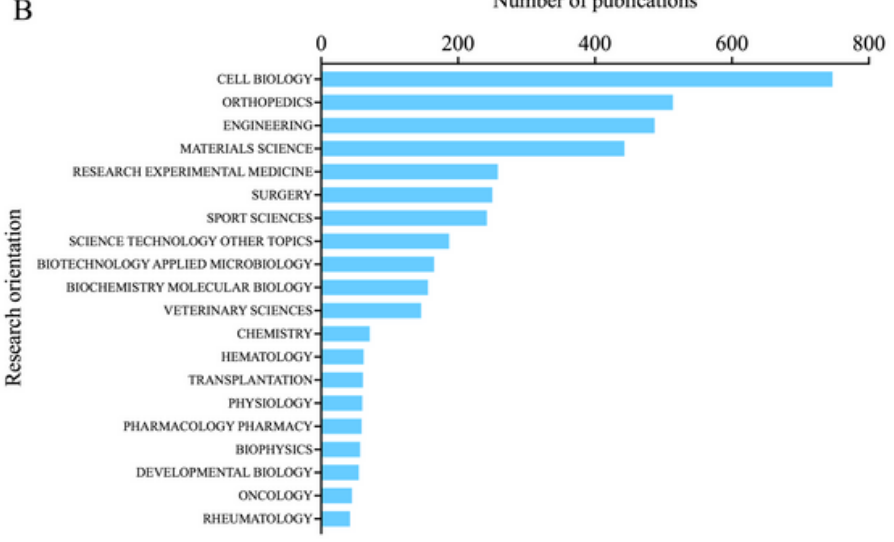

D

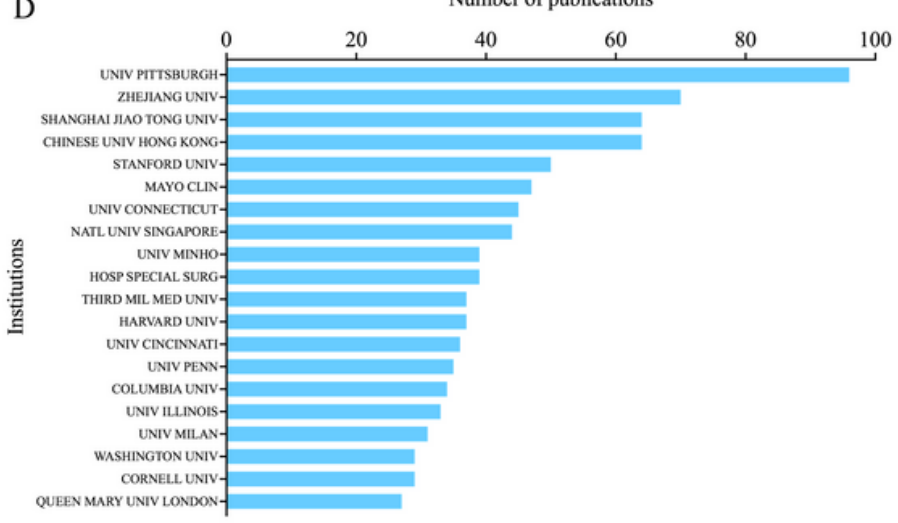

\section{Figure 3}

A The top 20 journals with most publications related to the research of stem cells for tendon. B The 20 research orientations and the number of publications in each orientation. $C$ The top 20 authors with most publications in this field. D The top 20 institutions of high-impact and the number of their publications. 
A

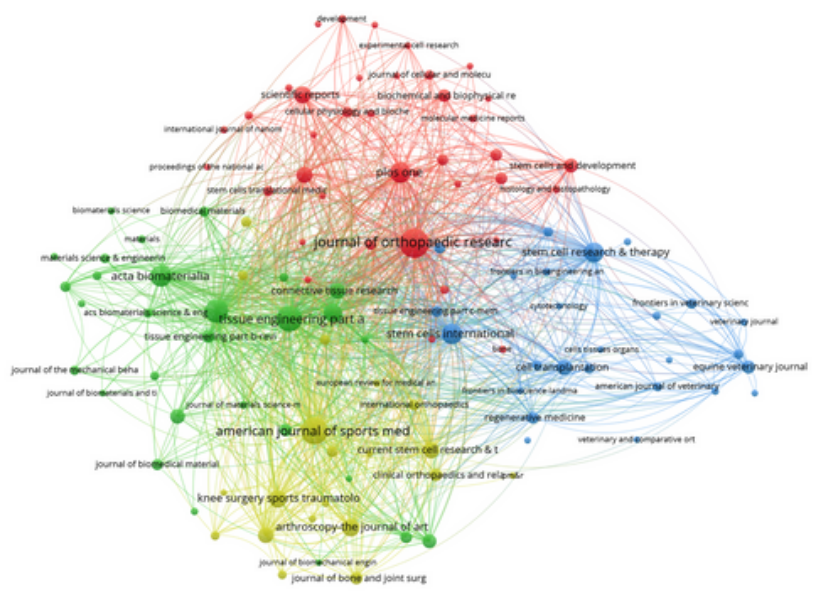

A vosiventer

B

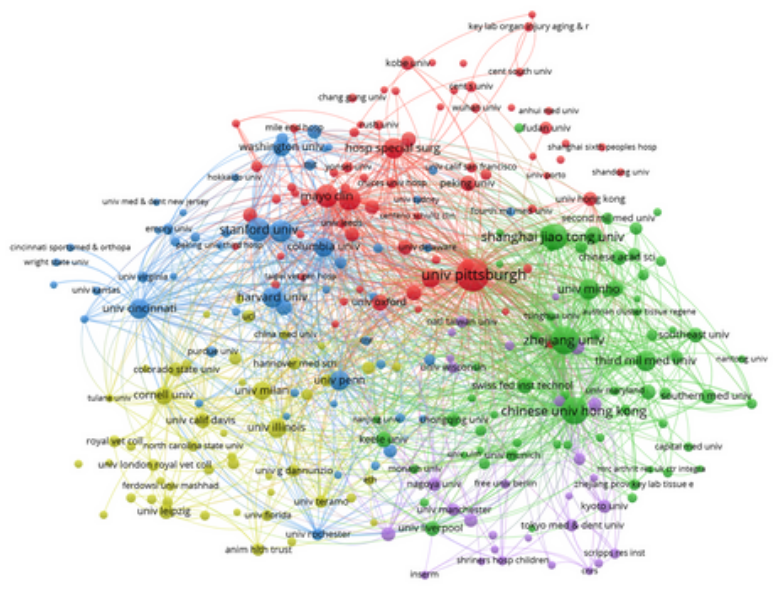

Av vosineser

C

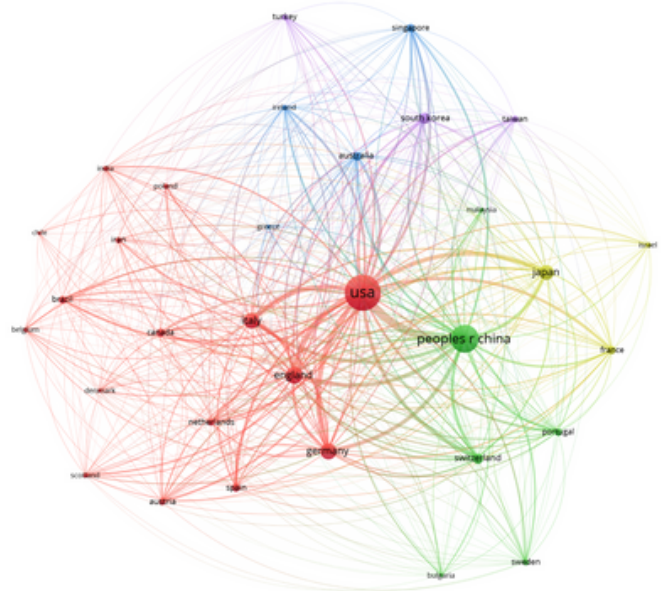

A vosinemer

\section{Figure 4}

Bibliographic coupling analysis of global research of stem cells for tendon. A Mapping on the 113 identified journals on the research of stem cells for tendon. B Mapping on 265 institutions in this field. C Mapping on the 32 countries in this field. The similarity relationship of two journals/institutions/countries was created by the line between two nodes in the figure. 
A

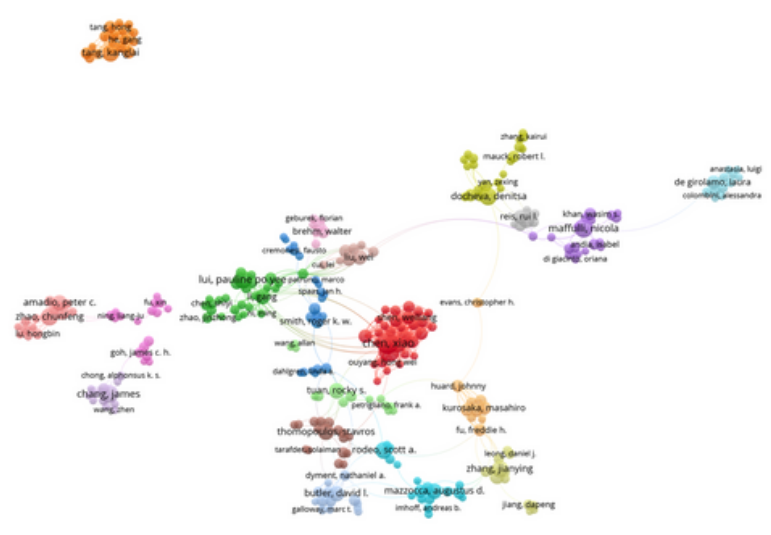

$\mathrm{C}$

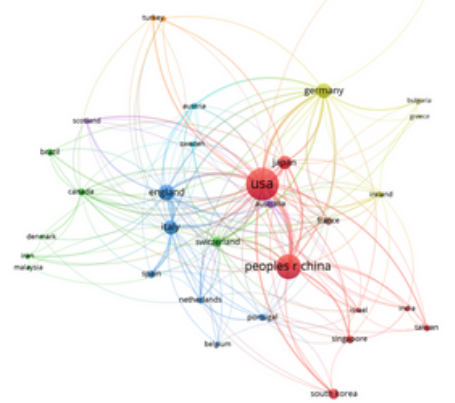

Avosivener

$\mathrm{E}$

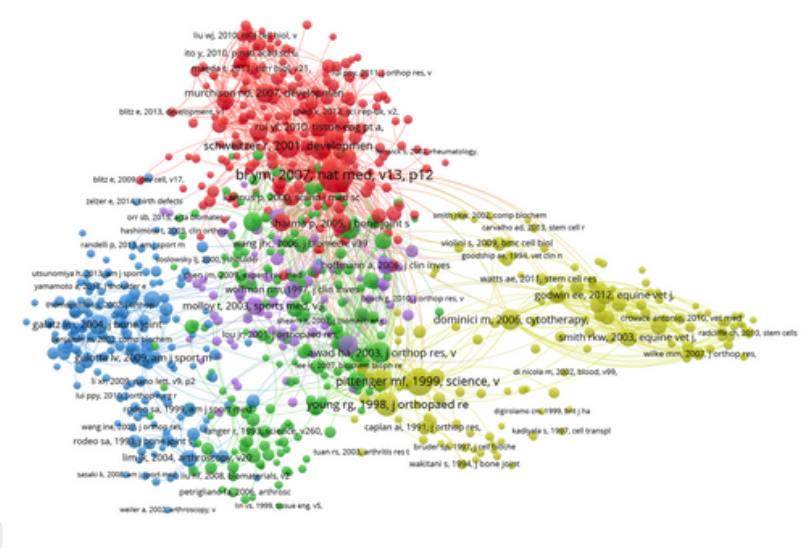

B

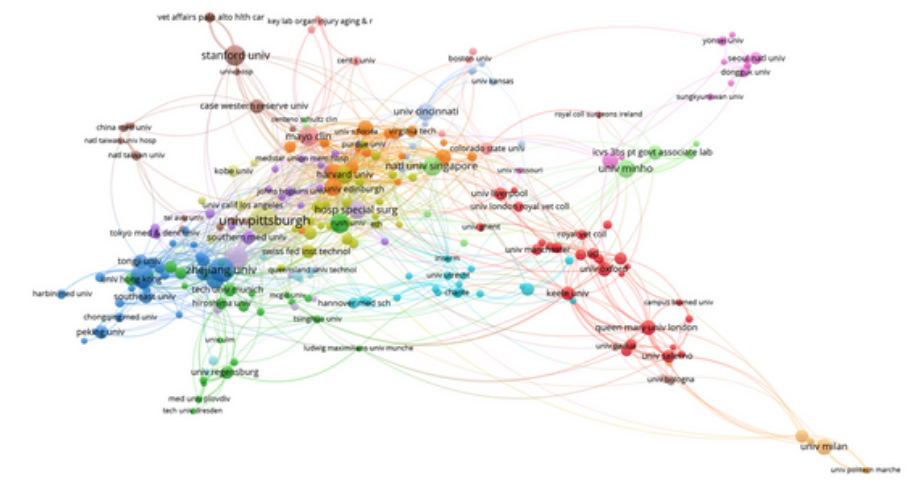
A. voswemer

D

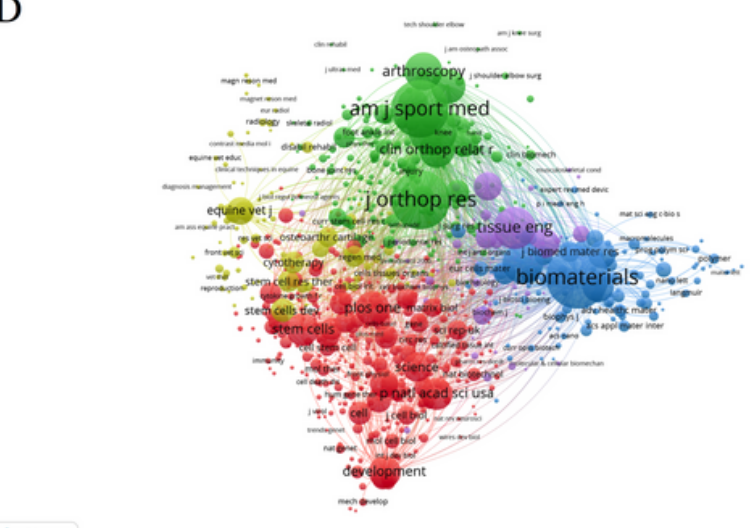

Figure 5

Co-authorship and co-citation analysis of global research of stem cells for tendon. A Mapping on the 378 identified authors by co-authorship analysis on the research of stem cells for tendon. B Mapping on the 265 identified institutions by co-authorship analysis in this field. C Mapping on the 32 identified countries by co-authorship analysis in this field. D Mapping on the 825 identified journals by co-citation analysis. $\mathrm{E}$ Mapping on the 808 identified references by co-citation analysis. 
Cluster 1: Animal study

A

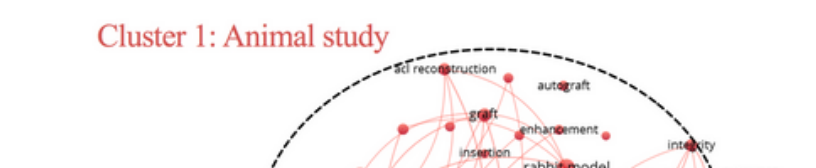

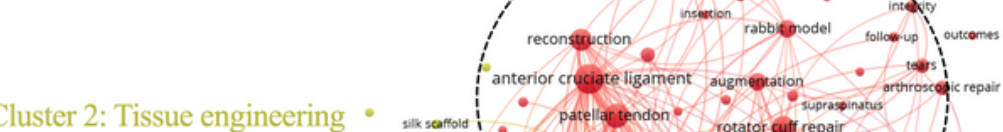

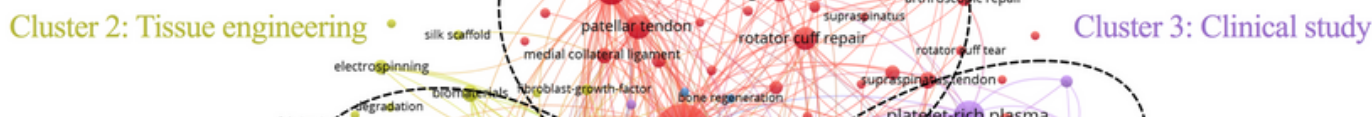

\& vosviewer

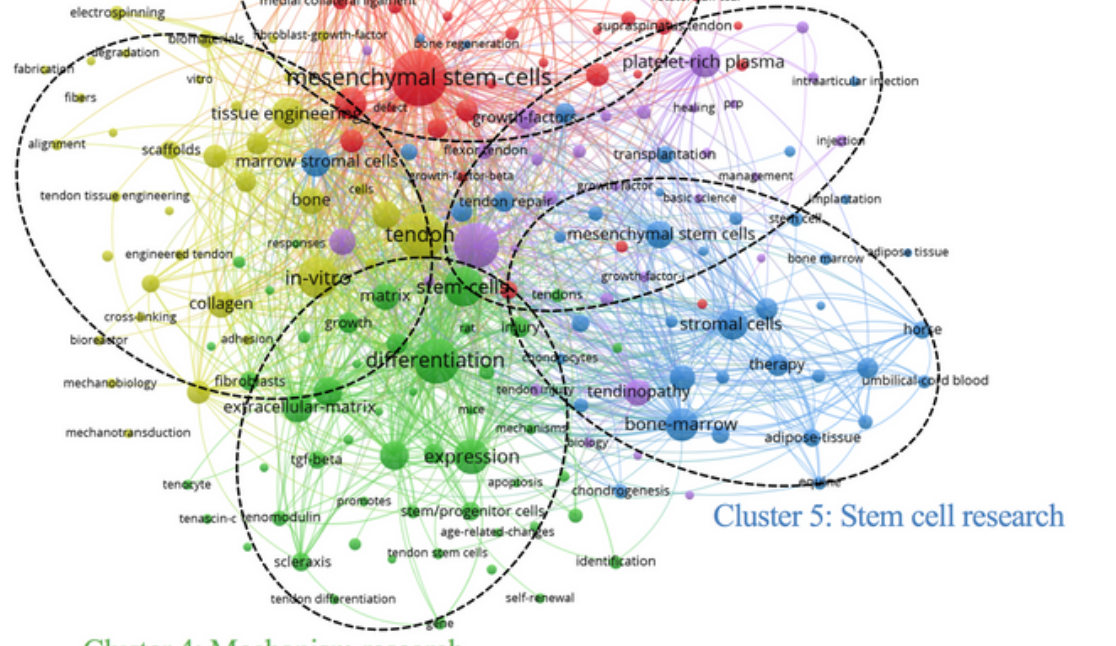

Cluster 4: Mechanism research

B

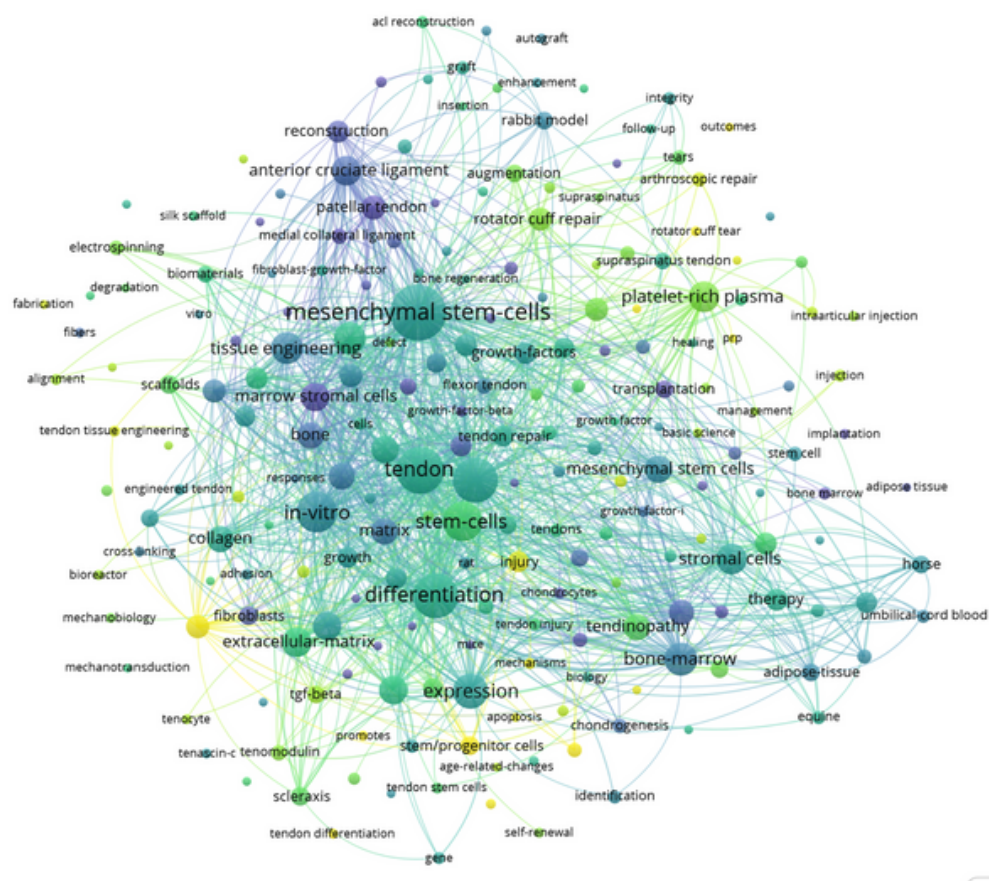

As vosviewer

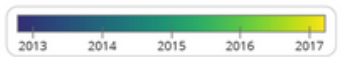

Figure 6

Co-occurrence analysis of global research of stem cells for tendon. A Mapping on keywords in the research of stem cells for tendon; the size of the nodes indicates the frequency, and the keywords are classified into five clusters: animal study (red color), tissue engineering (yellow color), mechanism research (green color), stem cell research (blue color) and clinical study (purple color). B Distribution of 
keywords based upon their time of appearance; purple keywords appeared earlier than green ones, and yellow keywords appear later than both of purple and green keywords. 